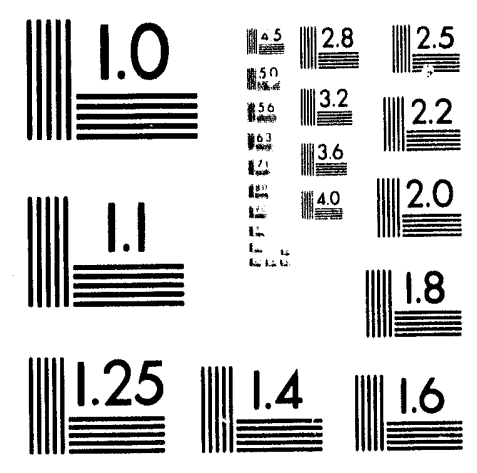



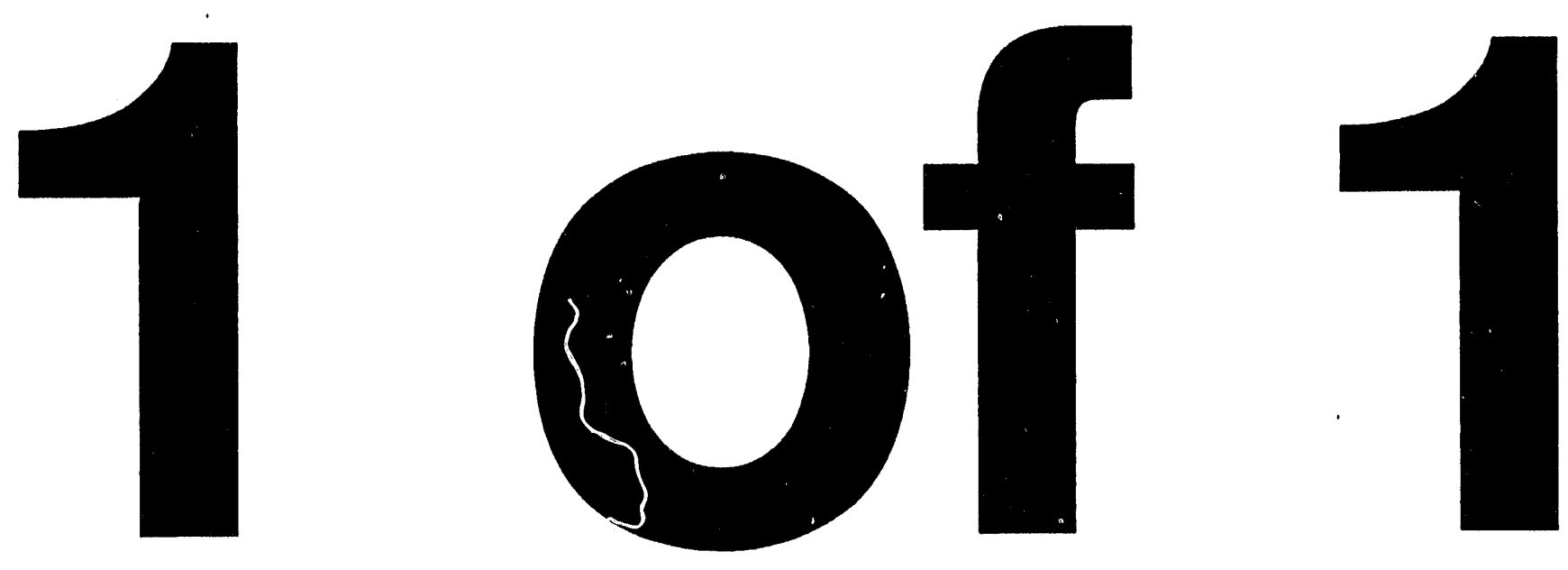


\section{$\cos f 9.209706-3$}

UCRL-JC-112168

PREPRINT

\section{Multifragment Source Lifetime in Intermediate Energy Heavy Ion Collisions}

T. C. Sangster, M. Begemann-Blaich, Th. Blaich, H. C. Britt, A. Elmaani, N. N. Ajitanand, M. N. Namboodiri

This paper was prepared for submittal to the Erice School Proceedings, "Progress in

Particle Nuclear Physics"

Erice, Sicily, Italy

September 8-15, 1992

November 1992

This is a preprint of a paper intended for publication in a journal or proceedings. Since changes may be made before publication, this preprint is made available with the understanding that it will not be cited or reproduced without the permission of the author.

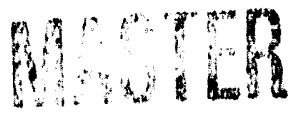




\section{DISCLAIMER}

This document was prepared as an account of work sponsored by an agency of the United States Government. Neither the United States Government nor the University of California nor any of their employees, makes any warranty, express or implied, or assumes any legal liability or responsibility for the accuracy, completeness, or usefulness of any information, apparatus, product, or process disclosed, or represents that its use would not infringe privately owned rights. Reference herein to any specific commercial products, process, or service by trade name, trademark, manufacturer, or otherwise, does not necessarily constitute or imply its endorsement, recommendation, or favoring by the United States Government or the University of California. The views and opinions of authors expressed herein do not necessarily state or reflect those of the United States Government or the University of Califomia, and shall not be used for advertising or product endorsement purposes. 


\title{
Multifragment Source Lifetime in Intermediate Energy Heavy Ion Collisions
}

\author{
T. C. Sangster ${ }^{1}$, M. Begemann-Blaich ${ }^{1,2}$, Th. Blaich ${ }^{1,3}$, H. C. Britt ${ }^{1}$, \\ A. Elmaani ${ }^{4}$, N. N. Ajitanand ${ }^{4}$ and M. N. Namboodiri ${ }^{1}$
}

1. Lawrence Livermore National Laboratury, Livermore, CA 94550

2. GSI, D-61 Darmstadt, Germany

3. Universitat Mainz, D-65 Mainz, Germany

4. State University of New York, Stonybrook, NY 11794

Complete two-fragment velocity sytematics have been measured over the full range of fragmentation products for the system ${ }^{56} \mathrm{Fe}+{ }^{197} \mathrm{Au}$ at 50 and $100 \mathrm{MeV} / \mathrm{A}$. Fragment emission lifetimes can be estimated using the technique of intensity interferometry [1-6] by comparing mixed-fragment $\left(Z_{1} \neq Z_{2}\right)$ reduced velocity [6] $\left(V_{\text {rel }} / \sqrt{Z_{1}+Z_{2}}\right)$ correlation functions with results from a classical three-body trajectory calculation in which the Coulomb influence and recoil effects of the emitting source are explicitly included. We have chosen to use the trajectory code $M E N E K A$ [7] for this comparison.

The data were taken at the Bevalac using the PAGODA [8] array. Fragments with velocities between 1.0 and $4.1 \mathrm{~cm} / \mathrm{ns}$ and charges between 5 and 53 were measured in eight identical composite detector modules arranged cylindrically around the target between polar angles of $22^{\circ}$ and $158^{\circ}$ on centerlines of $\pm 36^{\circ}, \pm 72^{\circ}$, $\pm 108^{\circ}$ and $\pm 144^{\circ}$. Each module subtended $\pm 13^{\circ}$ in and out of the beam plane. Details of the fragment identification algorithms and a summary of the inclusive IMF data can be found elsewhere [9-10].

All coincidence combinations in the measured charge interval $5 \leq Z_{i, j} \leq 53$ (exlcuding binary fission) were used to construct two-fragment correlation functions following the formalism of Ref. [11]. Examples of the reduced velocity correlation function are shown in Figure 1 for several constraints on the Coulomb product, $Z_{1} Z_{2}$. The solid lines are fits using a Fermi function which allows us to quantitatively assess differences in the correlation function shapes. Clearly there is a strong $Z$ dependence in the widths of the Coulomb holes; the three arrows show the half depth point of each hole.

The essential features of $M E N E K A$ are isotropic surface emission from a spherical source characterized by a unique radius parameter with angular momenta chosen randomly from ( 0 to $\left.l_{\max }\right)$. The emission energies are selected to reproduce the experimental data and the distribution of time delays between fragment emission is given by $e^{-t / \tau}$. The output was passed through the PAGODA acceptance filter and reduced velocity correlation functions were constructed for emission lifetimes of 50, 100, 250, 500 and $1000 \mathrm{fm} / \mathrm{c}$.

Figure 2 shows the comparison between the calculation and the ${ }^{56} \mathrm{Fe}+{ }^{197} \mathrm{Au}$ data. The $M E N E K A$ correlation functions have been fit with a modified Fermi distribution,

$$
f(x)=\frac{\operatorname{aexp}\left(\frac{d-x}{e}\right)}{1.0+\exp \left(\frac{b-x}{c}\right)},
$$

and these fits are compared with the data in Figure 2. If we compare the curves inside the Coulomb hole, then the emission lifetime appears to be less than $500 \mathrm{fm} / \mathrm{c}$ for both energies and somewhat less at 100 than at $50 \mathrm{MeV} / \mathrm{A}$. If we examine the half depth region, then the lifetime at the higher energy may well be less than $250 \mathrm{fm} / \mathrm{c}$. There is very little sensitivity for lifetimes less than about $250 \mathrm{fm} / \mathrm{c}$ and this is likely due to restrictions on the initial conditions for the three-body trajectory calculation.

The most striking feature of the MENEKA correlation functions is the enhancement at the edge of the Coulomb hole. The enhancement is most pronounced for the shorter lifetimes and decreases rapidly for lifetimes greater than $250 \mathrm{fm} / \mathrm{c}$. This suggests that it may be caused by the limited phase space available for absorbing small changes in $V_{\text {rel }}$ caused by the fragment-fragment Coulomb interaction. The dashed curves in Figure 1 are fits to Eq. (1) and clearly highlight the enhancement in the data. Although the enhancement is not as pronounced as in the calculation, it does appear to extend to considerably larger values of $V_{\text {red }}$ and to be independent of $Z_{1} Z_{2}$. Therefore, any measured enhancement in this region of the correlation function may be interpreted as an indication for very short emission lifetimes.

By measuring complete two-fragment velocity systematics, we have been able to estimate the source lifetime by comparing the data with the results of a classical three-body trajectory calculation in which the 
source lifetime is explicitly included. The comparison of reduced velocity correlation functions generated from a narrow range of $Z_{1} Z_{2}$ with the trajectory code $M E N E K A$ indicates that the emission lifetime may be less than $250 \mathrm{fm} / \mathrm{c}$. We have also shown that there is an enhancement in the correlation functions at the edge of the Coulomb hole which may be a further indication of very short emission times. This work was performed under the auspices of the US Department of Energy by the Lawrence Livermore National Laboratory under contract W-7405-ENG-48.

\section{References}

[1] Y. D. Kim et al., Phys. Rev. Lett. 67, 14 (1991).

[2] S. E. Koonin, Phys. Lett. 70B, 43 (1977).

[3] R. Trockel et al., Phys. Rev. Lett. $\underline{59}, 2844$ (1987).

[4] D. H. Boal et al., Rev. Mod. Phys. 62, 553 (1990).

[5] W. G. Gong et al., Phys. Rev. C43, 781 (1991); Phys. Rev. C45, 863 (1992).

[6] Y. D. Kim et al., Phys. Rev. C45, 387 (1992).

[7] A. Elmaani et al., Nucl. Instrum. Methods $A 313,401,(1992)$.

[8] M. M. Fowler et al., Nucl. Instrum. Methods A281, 517 (1989).

[9] M. Begemann-Blaich et al., Phys. Rev. C45, 677 (1992).

[10] T. C. Sangster et al., Phys. Rev. C, to be published, Oct. 1992.

[11] W. A. Zajc et al., Phys. Rev. C29, 2173 (1984).
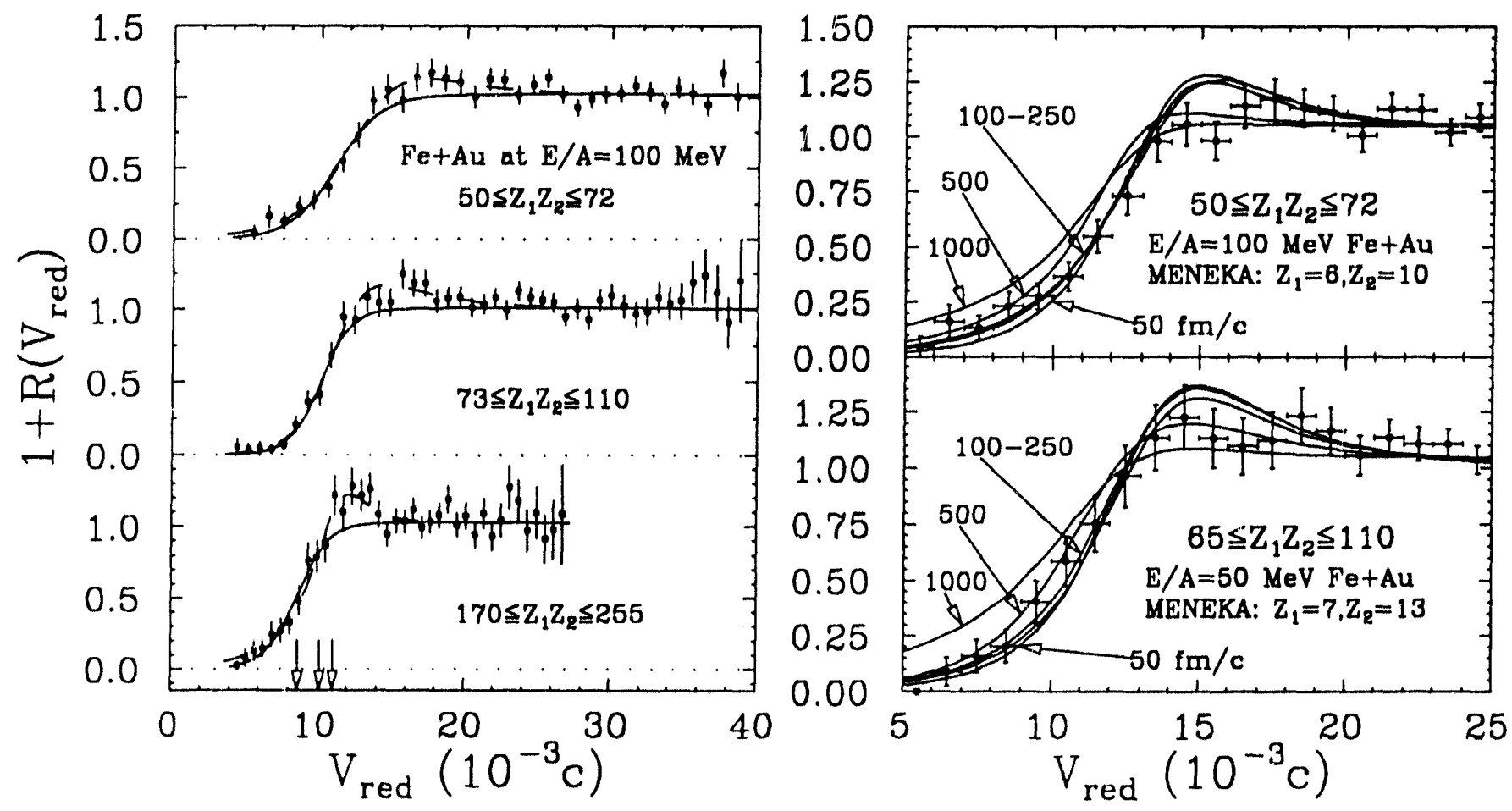

Figure 1: Mixed-fragment reduced velocity correlation functions for three different contraints on the Coulomb product, $Z_{1} Z_{2}$. The error bars indicate the statistical error only while the solid and dashed curves are, respectively, fits to the Fermi and modified Fermi (Eq. 1) distributions.

Figure 2: Comparison between the $V_{\text {red }}$ correlation functions generated with $M E N E K A$ for emission lifetimes from 50 to $1000 \mathrm{fm} / \mathrm{c}$ and the ${ }^{56} \mathrm{Fe}+{ }^{197} \mathrm{Au}$ data. 

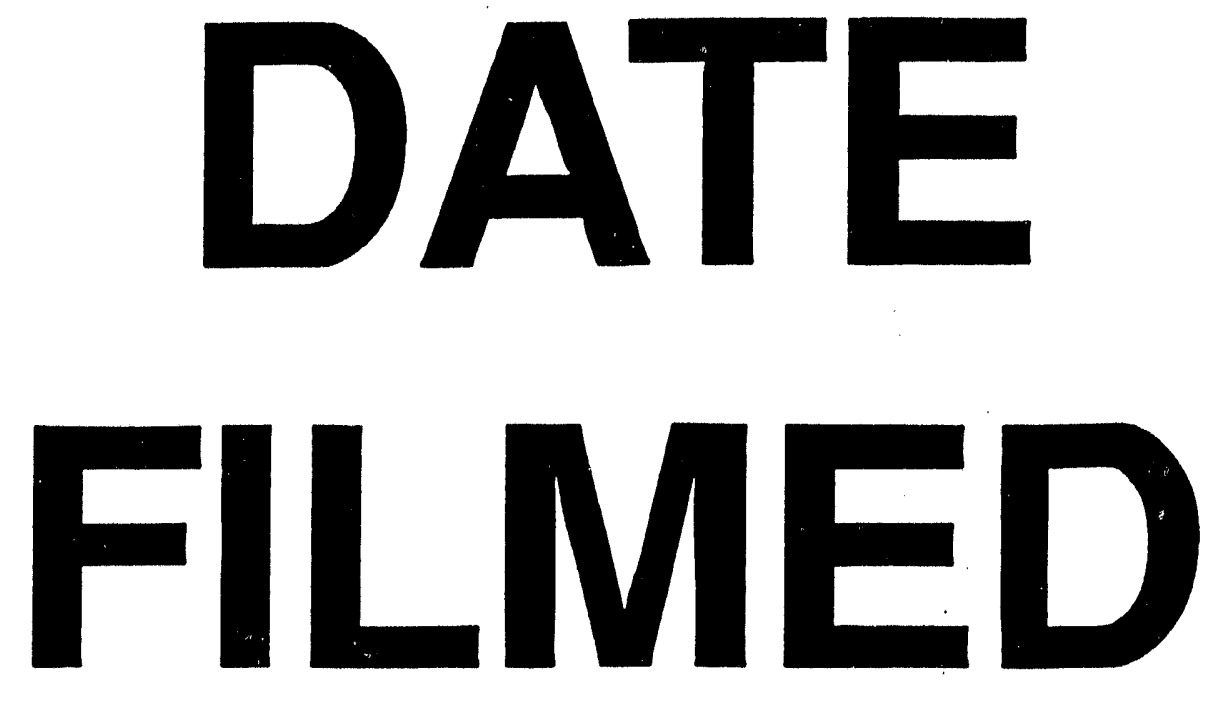

$11 / 18 / 93$
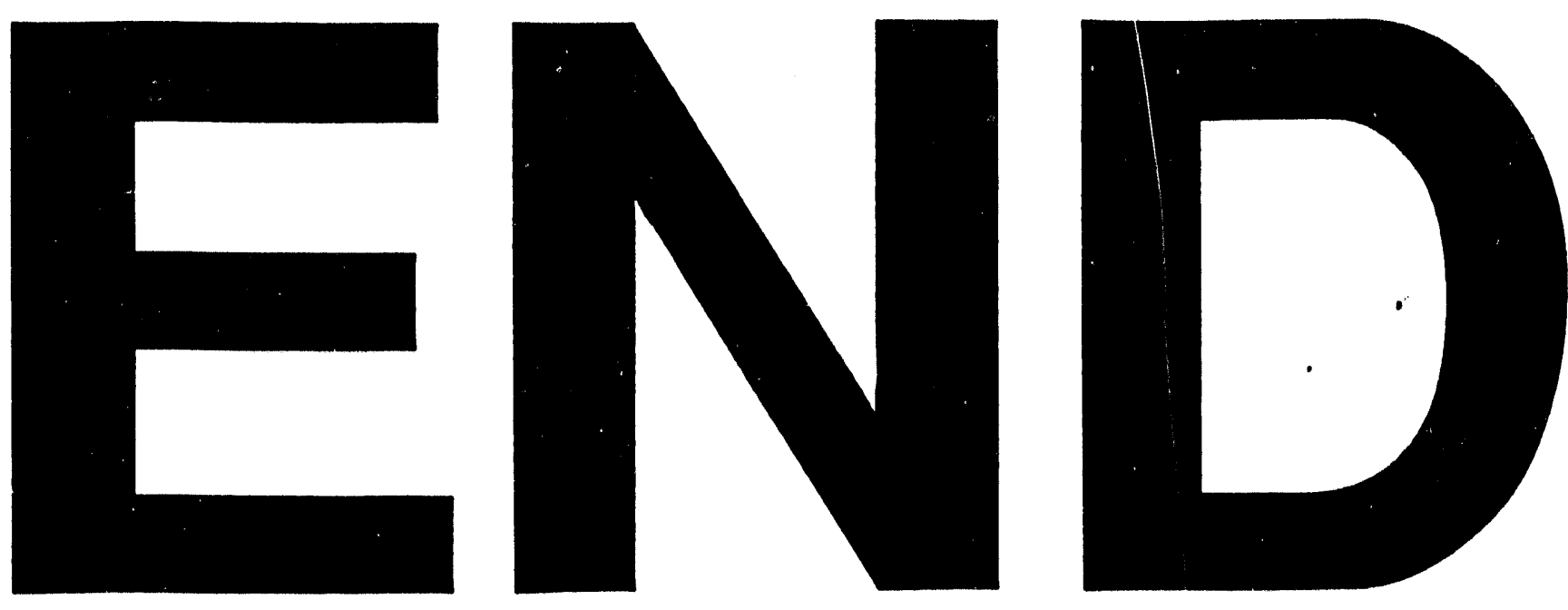
